\title{
An update on Müllerian-inhibiting substance: its potential application against ovarian cancer
}

\author{
Raymond R Y Wong ${ }^{1,{ }^{*}}$, Michael J Worley $\mathrm{Jr}^{2,{ }^{*}}$, Tony K H Chung ${ }^{1}$ and Yick Fu Wong ${ }^{1,2}$ \\ ${ }^{1}$ Department of Obstetrics and Gynaecology, The Chinese University of Hong Kong, Shatin, Hong Kong \\ ${ }^{2}$ Division of Gynecologic Oncology, Department of Obstetrics, Gynecology and Reproductive Biology, \\ Harvard Medical School, Brigham and Women's Hospital, Boston, Massachusetts, USA \\ ${ }^{*}$ ( R R Y Wong and M J Worley contributed equally to the paper as the first author)
}

Correspondence should be addressed to Y F Wong

Email yfwong@rics.bwh. harvard.edu

\begin{abstract}
Each year, 25000 women are newly diagnosed with ovarian cancer in the USA. The vast majority ( $>90 \%$ ) of cases are of epithelial origin. This highly lethal cancer carries a mortality rate of $>50 \%$ and a high risk of recurrence after conventional, first-line chemotherapy. Müllerian-inhibiting substance (MIS) is a gonadal hormone that causes regression of the Müllerian ducts. A series of studies have demonstrated that MIS also has multiple extraMüllerian functions including inhibition of epithelial ovarian cancer cells in vitro and in vivo. Accumulating evidence has shown that many human cancers are organized hierarchically and contain a small population of cancer stem cells (CSCs) that are inherently resistant to common chemotherapy and radiation therapy. The effect of MIS on ovarian CSC seems to be particularly useful in rescuing ovarian cancer patients with resistance to conventional treatment. Based on recent studies evaluating MIS, this review updates our current understanding of the molecular genetic aspects of MIS, its pathophysiology, as well as its potential to treat chemoresistant epithelial ovarian cancer.
\end{abstract}

\author{
Key Words \\ - Müllerian-inhibiting \\ substance \\ - ovarian cancer \\ - cancer stem cell
}

\section{Introduction}

In the USA, 25000 women are newly diagnosed with ovarian cancer each year (Jemal et al. 2009). Ovarian cancer is the second most common gynecologic cancer (Gershenson \& Ramirez 2012). In 2010 alone, ovarian cancer caused $\sim 14000$ deaths in the USA. While the overall 5-year survival rate for all cancers combined has improved, ovarian cancer has a poorer outcome, with a $47 \%$ survival rate. This is attributed to the fact that fewer than $25 \%$ of all ovarian cancer cases are detected at an early stage. Furthermore, the majority of advanced-stage ovarian cancer recurrences are extremely resistant to additional lines of chemotherapy (Shih \& Kurman 2005).
More than half a century ago, Alfred Jost resolved the controversy surrounding the mechanism of somatic sex differentiation by proving that male characteristics must be imposed on the fetus by the testicular hormones testosterone and Müllerian-inhibiting substance (MIS). Together, these hormones result in the virilization of the Wolffian ducts, urogenital sinus, and external genitalia, as well as the regression of the Müllerian ducts. If these hormones are absent or inactive, the fetus becomes phenotypically female (Josso 2008). Since the work of Jost, especially in the past three decades, the study of MIS has been expanded to its pathological significance and potential clinical application.

Published by Bioscientifica Ltd 
More than $90 \%$ of ovarian cancer cases are of epithelial histology and are believed to arise from the surface epithelium of the ovary (Quirk et al. 2005). As the ovarian surface epithelium also represents the origin of the Müllerian duct, the effect of MIS on the growth of ovarian cancer cells has been an area of intense study (Scully 1995).

The primary aim of this review was to provide a comprehensive update to our current understanding of the molecular genetic aspects of MIS, its pathophysiology, as well as its potential to treat epithelial ovarian cancer. Furthermore, the described in vitro and in vivo studies provide evidence as to the possible clinical application of MIS against epithelial ovarian cancer, particularly in the management of a chemoresistant disease.

\section{Molecular and genetic aspects of MIS}

Named after the German physiologist, Peter Müller, MIS has also been referred to as: Müllerian-inhibiting factor (MIF), Müllerian-inhibiting hormone, and anti-Müllerian hormone (AMH). MIS is a glycoprotein with a structure of a homodimer linked by disulfide, normally, produced by the Sertoli cells of the testis. MIS is a member of the transforming growth factor $\beta$ (TGF $\beta$ ) ligand superfamily. In humans, the gene for MIS is $A M H$ and is located on chromosome 19p13.3 with a size of 2960 bases. Human $A M H$ has five exons that code for a protein of 560 amino acids (Cate et al. 1986).

The MIS molecule must be proteolytically cleaved between R427 and S428 to generate the bioactive carboxy (C)-terminal fragment (Pepinsky et al. 1988, MacLaughlin et al. 1992). MIS is glycosylated at its amino (N)-terminus. While the functional significance of this is unknown, without the N-terminus to confer stability, the half of C-terminal MIS is markedly reduced in serum (Lane \& Donahoe 1998).

It is considered that the binding of MIS ligand to the MIS type II receptor, a serine threonine kinase, leads to heterodimerization with a type I receptor, initiating a signaling cascade (Baarends et al. 1994). The MIS type II receptor gene contains 11 exons and encodes a 63-kDa protein that is expressed at very high levels in the uterus, testis, and ovary. The MIS type II receptor is expressed in the cells surrounding the fetal Müllerian duct, in fetal gonads during the period of sex determination and Müllerian duct regression, and in pubertal and adult rat gonads. The type II receptor mRNA temporal and tissuespecific patterns of expression have been shown to match that of MIS (Teixeira et al. 1996). Several different type I receptors have been identified as interacting with the type II receptor (Alk3, Alk2, and Alk6). However, how each type I receptor interacts with the type II receptor in human tissues remains unknown.

\section{Pathophysiological features of MIS}

MIS has been known to have multiple functions. Among mammals, MIS inhibits the development of the Müllerian ducts into the uterus and fallopian tubes (Behringer 1994). In humans, these events occur during the first 8 weeks of gestation, and in the absence of MIS, the Müllerian structures develop. Simultaneously, the Wolffian ducts, responsible for the male reproductive structures, undergo programmed degeneration. Measurable blood levels of MIS vary by age and sex. MIS interacts with specific receptors located on the surface of target tissue cells. As described above, the most well characterized effect of MIS is the targeted degeneration of the fetal Müllerian ducts and is mediated through MIS type II receptors (Lee \& Donahoe 1993).

Among healthy females, MIS is detectable in small quantities, or not at all, within cord blood at birth. Levels of MIS undergo a dramatic increase by 3 months of age. Levels of MIS then begin to decline until 4 years of age and again rise until 8 years of age. MIS levels are unaffected by the onset of puberty and levels remain fairly constant until early adulthood. MIS levels in the blood slowly decline beginning at the age of 25 years and are typically undetectable at the onset of menopause. During the reproductive years, MIS is expressed by ovarian granulosa cells. MIS plays a key regulatory role in the formation of primary follicles through inhibition of excessive follicular recruitment by follicle-stimulating hormone. Owing to the role of MIS in folliculogenesis, MIS has become a useful marker to assess ovarian function in the setting of polycystic ovary syndrome and premature ovarian failure.

For healthy males, MIS is produced by the Sertoli cells of the testes and blood levels remain high throughout childhood. Blood levels of MIS then decline at the onset of puberty and remain low throughout adulthood. Studies have revealed that MIS influences the production of sex hormones and alterations in MIS levels may play a role in the onset of puberty for both males and females. Furthermore, MIS type II receptors have been identified in immature neurons of embryonic mice and are implicated in the development of gender-specific behaviors (Wang et al. 2009).

Evidence supports the concept that MIS indirectly causes the dissociation of the Müllerian duct epithelium in

Published by Bioscientifica Ltd. 
the rat by its effects on mesenchyme surrounding the Müllerian duct (Tsuji et al. 1992). This irreversible dissociation in the human fetus occurs by 51 days after ovulation (Taguchi et al. 1984). In the human male fetus, inadequate MIS activity can lead to the persistent Müllerian duct syndrome (PMDS), in which 46,XY males have normal external genitalia, but a rudimentary uterus and undescended testes. In PMDS, $A M H$ or the gene for its type II receptor (AMH-RII (AMHR2)) is usually mutated. MIS values have also proven to be a useful marker in the evaluation of the presence of testes and a measurement of testicular function among infants with ambiguous genitalia or those suffering from intersex conditions.

Comparing an individual's MIS level with average levels provides a guide to ovarian reserve and has proven useful for fertility assessment (Cupisti et al. 2007). In addition, it has the potential to rationalize the program of ovulation induction and decisions about the number of embryos to transfer in assisted reproduction techniques. Specifically, MIS levels can predict an excessive response in ovarian hyperstimulation with a sensitivity and specificity of 82 and $76 \%$ respectively. This may allow providers to maximize the likelihood of pregnancy, while minimizing the risk of developing ovarian hyperstimulation syndrome (Broer et al. 2011).

\section{MIS inhibits epithelial ovarian cancer in vitro and in vivo}

As mentioned previously, epithelial ovarian carcinomas are the most common form of ovarian carcinoma and arise from coelomic epithelium. Similarly, the Müllerian ducts also derive from invaginated coelomic epithelium. As MIS plays a key role in the regression of the fetal Müllerian structures, Donahoe et al. $(1979,1981)$ proposed that MIS could inhibit tumors of Müllerian origin. Since this hypothesis, purified MIS has reproducibly demonstrated antiproliferative effects against epithelial ovarian cancer in vitro and in vivo.

Purified MIS inhibits several human ovarian cancer cell lines of Müllerian origin in vitro. In addition, the MIS type II receptor is expressed in these cell lines and in the ascites cells of patients with ovarian cancer, providing additional evidence that human ovarian cancer may be a target for MIS as a potential chemotherapeutic agent (Stephen et al. 2002).

In a report by Stephen et al. (2002), highly purified MIS successfully inhibited ovarian cancer in vivo. Specifically, immunosuppressed mice were treated with MIS after implanting OVCAR8 or IGROV1 human ovarian cancer cells beneath the renal capsules. Mice were given daily injections of $10 \mu \mathrm{g}$ purified exogenous recombinant human MIS (rhMIS) or via endogenous MIS secreted from cells growing on biodegradable mesh. After treatment, the average graft size ratio (change in size compared with starting size) of the OVCAR8 tumor implants was larger in the control mice, when compared with mice treated with parenteral MIS for 2 weeks $(P<0.019)$ or 3 weeks $(P<0.001)$. Similarly, animals treated with MIS produced from transfected cells impregnated on a biodegradable mesh also experienced smaller tumor implants $(P=0.02)$. The average graft size ratio of the IGROV1 tumors was larger in the control animals than in those treated with injected MIS $(P=0.0174)$. These findings indicate that highly purified MIS, delivered parenterally, or MIS produced endogenously, results in inhibition of human ovarian cancer cell lines in vivo. Based on preclinical data collected so far, it would appear appropriate to evaluate the role of MIS as an adjuvant in ovarian cancer therapy in the setting of a clinical trial.

To examine whether rhMIS can be used as a nontoxic, naturally occurring, growth inhibitor and as an effective anticancer drug in long-term studies, rhMIS was tested against mouse ovarian carcinoma MOVCAR cells in growth inhibition assays in vitro, and in vivo among 6-week-old female nude mice. Results showed that rhMIS significantly inhibited MOVCAR cell growth in vitro and in vivo measured at weekly intervals for up to 20 weeks. These results suggest that rhMIS might be used safely and effectively to treat ovarian malignancies as significant inhibition of tumor growth was observed without obvious toxicity. Furthermore, evidence exists that human ovarian ascites cells from a large percentage of patients with stage III/IV ovarian epithelial cancer bind to rhMIS and their growth is inhibited by MIS (Lane \& Donahoe 1998). As a result of these studies, clinical trials may be planned to study the efficacy of MIS against epithelial ovarian tumors in humans when Good Manufacturing Practices for preparations of rhMIS are available (Pieretti-Vanmarcke et al. 2006).

Developing therapeutics capable of both inhibiting tumor growth and preventing invasion, contributing to migration and metastases, could be beneficial. Chang et al. (2011) designed in vitro and in vivo experiments to determine if MIS inhibited the movement of cancer lines IGROV1 (ovarian epithelial carcinoma), HEp3 (epithelioid head and neck carcinoma), MDA-MB-231 (breast epithelial carcinoma), and HT1080 (highly metastatic fibrosarcoma) in cell culture invasion/migration chamber assays and in chick embryo metastasis assays. Results showed that MIS,

Published by Bioscientifica Ltd. 
at concentrations below those that inhibited cell proliferation, blocked in vitro invasion and in vivo migration of epithelial cancer cells that express the MIS receptor. These findings suggest that MIS may be as effective as an antimetastatic agent as it is as an anti-proliferation agent. This ability to target more than one aspect of cancer biology is predicted to make MIS an advantageous anticancer agent in the clinical setting (Masiakos et al. 1999).

\section{Mechanism of MIS-mediated growth inhibition in ovarian cancer cells}

To test whether rhMIS acts via its receptor, Masiakos et al. studied six human ovarian cancer cell lines and samples of ascites cells isolated from 27 patients with stage III or IV ovarian serous carcinoma. Production of rhMIS was scaled up and rhMIS was labeled with biotin for binding studies, cloned with the human MIS type II receptor for mRNA detection, and antibodies to an extracellular domain peptide were raised for protein detection. These probes were first tested on ovarian cancer cell lines and then applied to primary ovarian ascites cells. The results showed that rhMIS inhibited colony growth of five of six cell lines that expressed the human MIS type II receptor mRNA by northern analysis, while not inhibiting receptor-negative COS cells. Flow cytometry performed on MIS-sensitive ovarian cancer cell lines demonstrated specific and saturable binding of rhMIS $\left(K_{\mathrm{d}}=10.2 \mathrm{~nm}\right)$. Ascites cells from 15 out of 27 (56\%) patients tested bound to biotinylated MIS (MIS-biotin) and out of the 11 ascites cells that grew in soft agarose, 9 (82\%) showed statistically significant inhibition of colony formation. Of the 15 patients whose ascites cells bound to MIS-biotin, mRNA was available for analysis from nine patients, and ascites cells from 8 (88.9\%) of them expressed MIS type II receptor mRNA by RT-PCR, showing a significant correlation compared with binding $(P=0.025)$. Solid ovarian cancers were positive for the MIS type II receptor protein by immunohistochemical staining, which colocalized with staining for antibody to CA-125 (OC-125). These results suggest that the detection of the MIS type II receptor by flow cytometry may be a useful predictor of therapeutic response to MIS and may be a modality to rapidly choose patients with late-stage ovarian cancer for treatment with MIS (Masiakos et al. 1999).

To uncover the molecular mechanism by which MIS inhibits the growth of ovarian cancer cells, Ha et al. analyzed the MIS-sensitive human ovarian cancer cell line OVCAR8, which expresses significant levels of MIS type II receptor. Experiments demonstrated that the growth of the OVCAR8 cells, which express MIS type II receptor mRNA, was inhibited following the expression of bioactive MIS ligand. MIS-induced apoptosis of OVCAR8 cells was detected by Annexin V-FITC staining. Fluorescenceactivated cell sorting showed a $14-18 \%$ increase in the G1 phase of the OVCAR8 cells after $48 \mathrm{~h}$ of treatment with MIS. MIS-induced p16 (CDKN2A) expression was mediated through the endogenous MIS type II receptor while MIS-induced p16 abrogated cell growth. MIS also increased the level of E2F1 protein in OVCAR8 cells, reflecting a mechanism mediated by TGF $\beta$ that decreased E2F1 mRNA and protein level during growth inhibition. The increase in E2F1 level by MIS may result from a decrease in p130 (RBL2) level and reversal of E2F4- or E2F5-p130-mediated repression of the E2F1 promoter. The growth inhibitory effect of E2F1 overexpression on OVCAR8 cell growth might be enhanced by the lack of RB (RB1) expression in this cell line. It seems that MIS-treated cells accumulated in the G1 phase of the cell cycle and subsequently underwent apoptosis. MIS up-regulated the cyclin-dependent kinase inhibitor p16 through an MIS type II receptor-mediated mechanism and inhibited growth in the absence of detectable or inactive RB protein. Prolonged treatment with MIS down-regulated the RB-related protein p130 and increased the RB familyregulated transcription factor E2F1, overexpression of which inhibited growth. These findings demonstrate that p16 is required for MIS-mediated growth inhibition in ovarian epithelial cancer cells and suggest that up-regulation of E2F1 also plays a role in this process (Ha et al. 2000). Thus, up-regulation of p16 protein by either gene transfer technique or MIS treatment could be therapeutically beneficial in treatment of ovarian cancer patients.

\section{MIS and ovarian cancer stem cells}

Growing evidence supports the concept that cancer is a stem cell disease. To assist future investigation, it has become vital to successfully identify cancer stem/progenitor cells. Once identified and isolated, specific therapies can be developed that target this population of cells. Several studies support the hypothesis that cancer stem cells (CSCs) facilitate resistance to chemotherapeutic agents as well as play an integral role in tumor growth and metastases.

'Side population' (SP) cells, identified by Hoechst 33342 dye exclusion in a wide range of cancers, appear to be enriched for CSCs. Szotek et al. reported that SP cells form larger tumors and have higher tumorigenic

Published by Bioscientifica Ltd. 
propensity than do non-SP (NSP) cells in mouse ovarian cancer cell lines (MOVCAR7). The stem cell population was inhibited by MIS. By contrast, NSP cells were more significantly inhibited by the chemotherapeutic agent doxorubicin (Szotek et al. 2006). These findings suggest that chemotherapeutic agents and MIS may differentially affect populations in human ovarian cancer, which are relatively chemoresistant and demonstrate stem cell characteristics. Specifically, traditional chemotherapeutic agents appear to inhibit the NSP cells but enable enrichment of SP cells containing stem/progenitor cells. By contrast, MIS primarily inhibits the stem/progenitor cell-enriched population. Future studies would benefit from therapies directed at both SP and NSP cells to assure eradication of both cell populations.

As mentioned before, it is essential to successfully identify the stem cell population to allow for future investigation. Wei et al. selected a panel of markers from 95 human cell surface antigens shared among human ovarian primary cancers, ovarian cancer cell lines, and normal fimbria. A panel consisting of CD44, CD24, and EPCAM appeared to select cells that most readily formed colonies. Cells expressing CD44, CD24, and EPCAM also exhibited stem cell characteristics including: shorter tumor-free intervals in vivo, and enhanced migration in invasion assays in vitro. Again, conventional chemotherapeutic agents (i.e. doxorubicin, cisplatin, and paclitaxel) increased this population enriched for tumor-initiating cells, which conversely was inhibited by MIS or SP600125, an anthrapyrazolone that is a small molecule acting as a MIS agonist in MIS type II receptor (Wei et al. 2010). Apart from the potential therapeutic benefits displayed in these studies, these findings also suggest that this population is amenable to isolation by flow cytometry. Once isolated, SP cells could potentially be screened for novel treatment strategies including targeted agents such as MIS.

As the MISRII (AMHR2)-expressing surface epithelium of the ovary is normally characterized by expression of epithelial and mesenchymal markers, which become predominantly mesenchymal in transgenic animals as tumor initiation occurs, Meirelles et al. refined the $3^{+}$ population in ovarian cancer cell lines by negative selection for E-cadherin $\left(3^{+} \mathrm{Ecad}^{-}\right)$, down-regulation of which occurs during epithelial-to-mesenchymal transformation in a variety of cancers and is associated with poor outcome. The results showed that $3^{+} \mathrm{Ecad}^{-}$are more tumorigenic than either $3^{+}$cells alone or triple-negative cells that retain expression of ECAD. MIS inhibits whereas doxorubicin stimulates colony and monolayer growth of the stem cell-enriched population $\left(3^{+} \mathrm{Ecad}^{-}\right)$. A cell cycle study demonstrated that MIS inhibits ovarian cancer cells by inducing $\mathrm{G} 1$ arrest of the $3^{+} \mathrm{Ecad}^{-}$subpopulation through the induction of cyclin-dependent kinase inhibitors (Meirelles et al. 2012). These results suggest that chemotherapeutics may be stimulative to CSCs and that selective inhibition of these cells by treating with MIS should be considered in the development of chemoresistance therapeutics. If the poor response to chemotherapeutic agents and the favorable response to MIS can be repeated in the stem population isolated from a significant number of patients, then it appears appropriate to recommend that treatment of ovarian cancers should be changed to include sensitivity testing of all of the heterogeneous populations when the diagnosis is made.

\section{Source of MIS for preclinical and clinical use}

If MIS has to be used for therapeutic purposes, an inexpensive and reliable source must be found. Taking this into consideration, some studies have sought to produce MIS in plants. Delivery systems take on increased importance, as the therapeutic must be the complex ligand, which has the advantage of receptor specificity. Based on the principles of tissue engineering, a biodegradable mesh can be utilized as a scaffold for creation of neoorgans made from cells transfected with the gene for MIS $(A M H)$. This would enable continuous in vivo production of the MIS protein.

When cells are transfected with genomic MIS, they can produce and secrete MIS into media, where MIS can be quantified by ELISA technique. Cells may be seeded onto a biodegradable mesh with subsequent implantation into immunosuppressed mice. Increasing levels of MIS production can be detected in the serum of mice. When human ovarian tumors were implanted beneath the renal capsule of these mice, growth of the tumor was significantly suppressed. It is the ultimate goal of these experiments to seed a patient's own cells onto the mesh after in vitro transfection of the cells with the gene for MIS (AMH; Teixeira et al. 2001). Originally, a clone MIS was used to produce rhMIS for research. Recently, a recombinant, internally FLAG-tagged form of hMIS with the tag (DYKDDDDK) immediately after the cleavage site (427-428) of MIS at the C-terminus with a modified dibasic cleavage motif sequence has been expressed. This construct results in a highly pure, endogenously processed (cleaved) FLAG MIS, that is able to bind and affinity purify both transfected and endogenous MIS type II receptor. The availability of this fully functional, epitope-tagged form of MIS should facilitate scale-up for preclinical and clinical

Published by Bioscientifica Ltd. 
use in the future (Papakostas et al. 2010). It is noted that widespread clinical use of MIS may await the availability of an international standard for MIS, so that results obtained from different settings may be reliably compared.

\section{Summary}

A series of studies have supported MIS as a promising therapy for epithelial ovarian cancer and warrant further clinical evaluation of MIS for the management of this devastating gynecologic malignancy. Evidence for cancer chemoresistance posits that cytotoxic survival of a subpopulation of tumor progenitors (so-called CSCs) drives the propagation of recurrent malignant disease, underscoring the need for new therapeutics that target such primitive cells. The discoveries of MIS targeting on chemoresistant CSC have created new opportunities to develop a personalized adjunct treatment for epithelial ovarian cancer.

\section{Declaration of interest}

The authors declare that there is no conflict of interest that could be perceived as prejudicing the impartiality of the review.

\section{Funding}

This review did not receive any specific grant from any funding agency in the public, commercial or not-for-profit sector.

\section{Acknowledgements}

The author Raymond Wong specially thanks Marshall K Bartlett Prof. Patricia K Donahoe's supervision of research on MIS at a summer college student program, the Pediatric Surgery Laboratories, Harvard Medical School, Massachusetts General Hospital (Boston, MA, USA), and Dr Leo Andrew Benedict's suggestions to the manuscript.

\section{References}

Baarends WM, van Helmond MJ, Post M, van de Schoot PJ, Hoogerbugge JW, de Wine JP, Uilenboek JT, Karels B, Wilming LG, Mejijes JH et al. 1994 A novel member of the transmembrane serine/threonine kinase receptor family is specifically expressed in the gonads and in mesenchymal cells adjacent to the Müllerian duct. Development 12 189-197.

Behringer RR 1994 The in vivo roles of Müllerian-inhibiting substance. Current Topics in Development Biology 29 171-187.

Broer SL, Dólleman M, Opmeer BC, Fauser BC, Mol BW \& Broekmans FJ $2011 \mathrm{AMH}$ and AFC as predictors of excessive response in controlled ovarian hyperstimulation: a meta-analysis. Human Reproduction Update 17 46-54. (doi:10.1093/humupd/dmq034)

Cate RL, Mattaliano RJ, Hession C, Tizard R, Farber NM, Cheung A Ninfa EG, Frey AZ, Gash DJ, Chow EP et al. 1986 Isolation of the bovine and human genes for Müllerian inhibiting substance and expression of the human gene in animal cells. Cell 45 685-698. (doi:10.1016/00928674(86)90783-X)

Chang HL, Pieretti-Vanmarcke R, Nicolaou F, Li X, Wei X, MacLaughlin DT \& Donahoe PK 2011 Müllerian inhibiting substance inhibits invasion and migration of epithelial cancer cell lines. Gynecologic Oncology 120 128-134. (doi:10.1016/j.ygyno.2010.09.017)

Cupisti S, Dittrich R, Mueller A, Strick R, Stiegler E, Binder H, Beckmann MW \& Strissel P 2007 Correlations between anti-Müllerian hormone, inhibin B, and activin A in follicular fluid in IVF/ICSI patients for assessing the maturation and developmental potential of oocytes. European Journal of Medical Research 12 604-608.

Donahoe PK, Swann DA, Hayashi A \& Sullivan MD 1979 Müllerian duct regression in the embryo correlated with cytotoxic activity against human ovarian cancer. Science 205 913-915. (doi:10.1126/science. 472712)

Donahoe PK, Fuller AF Jr, Scully RE, Guy SR \& Budzik GP 1981 Müllerian inhibiting substance inhibits growth of a human ovarian cancer in nude mice. Annals of Surgery 194 472-480. (doi:10.1097/00000658198110000-00010)

Gershenson DM \& Ramirez PT 2012 Ovarian cancer In Gynecology and Obstetrics. Whitehouse Station, NJ, USA: Merck Sharp \& Dohme Corp. (available at: http://www.merckmanuals.com/professional/ gynecology_and_obstetrics/gynecologic_tumors/ovarian_cancer.html)

Ha TU, Segev DL, Barbie D, Masiakos PT, Tran TT, Dombkowski D, Glander M, Clarke TR, Lorenzo HK, Donahoe PK et al. 2000 Müllerian inhibiting substance inhibits ovarian cell growth through an rb-independent mechanism. Journal of Biological Chemistry 275 37101-37109. (doi:10.1074/jbc.M005701200)

Jemal A, Siegel R, Ward E, Hao Y, Xu J \& Thun MJ 2009 Cancer statistics. CA: A Cancer Journal for Clinicians 59 225-249. (doi:10.3322/caac. 20006)

Josso N 2008 Professor Alfred Jost: the builder of modern sex differentiation. Sexual Development 2 55-63. (doi:10.1159/000129690)

Lane AH \& Donahoe PK 1998 New insights into Müllerian inhibiting substance and its mechanism of action. Journal of Endocrinology $\mathbf{1 5 8}$ 1-6. (doi:10.1677/joe.0.1580001)

Lee MM \& Donahoe PK 1993 Müllerian inhibiting substance: a gonadal hormone with multiple functions. Endocrine Reviews 14 152-164.

MacLaughlin DT, Hudson PL, Graciano AL, Kenneally MK, Ragin RC, Manganaro TF \& Donahoe PK 1992 Müllerian duct regression and antiproliferative bioactivities of Müllerian inhibiting substance reside in its carboxy-terminal domain. Endocrinology 131 291-296.

Masiakos PT, MacLaughlin DT, Maheswaran S, Teixeira J, Fuller AF Jr, Shah PC, Kehas DJ, Kenneally MK, Dombkowski DM, Ha TU et al. 1999 Human ovarian cancer, cell lines, and primary ascites cells express the human Müllerian inhibiting substance (MIS) type II receptor, bind, and are responsive to MIS. Clinical Cancer Research 5 3488-3499.

Meirelles K, Benedict LA, Dombkowski D et al. 2012 Human ovarian cancer stem/progenitor cells are stimulated by doxorubicin but inhibited by Müllerian inhibiting substance. PNAS 109 2358-2363. (doi:10.1073/ pnas.1120733109)

Papakostas TD, Pieretti-Vanmarcke R, Nicolaou F, Thanos A, Trichonas G, Koufomichali X, Anago K, Donahoe PK, Teixeira J, MacLaughlin DT et al. 2010 Development of an efficiently cleaved, bioactive, highly pure FLAG-tagged recombinant human Müllerian inhibiting substance. Protein Expression and Purification 70 32-38. (doi:10.1016/j.pep. 2009. 09.004)

Pepinsky RB, Sinclair LK, Chow EP, Mattaliano RJ, Manganaro TF, Donahoe PK \& Cate RL 1988 Proteolytic processing of Müllerian inhibiting substance produces a transforming growth factor- $\beta$-like fragment. Journal of Biological Chemistry 263 18961-18964.

Pieretti-Vanmarcke R, Donahoe PK, Szotek P, Manganaro T, Lorenzen MK, Lorenzen J, Connolly DC, Halpern EF \& MacLaughlin DT 2006 Recombinant human Müllerian inhibiting substance inhibits longterm growth of MIS type II receptor-directed transgenic mouse ovarian 
cancers in vivo. Clinical Cancer Research 12 1593-1598. (doi:10.1158/ 1078-0432.CCR-05-2108)

Quirk JT, Natarajan N \& Mettlin CJ 2005 Age-specific ovarian cancer incidence rate patterns in the united states. Gynecologic Oncology 99 248-250. (doi:10.1016/j.ygyno.2005.06.052)

Scully RE 1995 Pathology of ovarian cancer precursors. Journal of Cellular Biochemistry. Supplement 23 208-218. (doi:10.1002/jcb. 240590928)

Shih I \& Kurman RJ 2005 Molecular pathogenesis of ovarian borderline tumors: new insights and old challenges. Clinical Cancer Research $\mathbf{1 1}$ 7273-7279. (doi:10.1158/1078-0432.CCR-05-0755)

Stephen AE, Pearsall LA, Christian BP, Donahoe PK, Vacanti JP \& MacLaughlin DT 2002 Highly purified Müllerian inhibiting substance inhibits human ovarian cancer in vivo. Clinical Cancer Research 8 2640-2646.

Szotek PP, Pieretti-Vanmarcke R, Masiakos PT, Dinulescu DM, Connolly D, Foster R, Dombkowski D, Preffer F, Maclaughlin DT \& Donahoe PK 2006 Ovarian cancer side population defines cells with stem cell-like characteristics and Müllerian inhibiting substance responsiveness. PNAS 103 11154-11159. (doi:10.1073/pnas.0603672103)

Taguchi O, Cunha GR, Lawrence WD \& Robboy SJ 1984 Timing and irreversibility of Müllerian duct inhibition in the embryonic reproductive tract of the human male. Developmental Biology 106 394-398. (doi:10.1016/0012-1606(84)90238-0)

Teixeira J, He WW, Shah PC, Morikawa N, Lee MM, Catlin EA, Hudson PL, Wing J, Maclaughlin DT \& Donahoe PK 1996 Developmental expression of a candidate Müllerian inhibiting substance type II receptor. Endocrinology 137 160-165.

Teixeira J, Maheswaran S \& Donahoe PK 2001 Müllerian inhibiting substance: an instructive developmental hormone with diagnostic and possible therapeutic applications. Endocrine Reviews 22 657-674.

Tsuji M, Shima H, Yonemura CY, Brody J, Donahoe PK \& Cunha GR 1992 Effect of human recombinant Müllerian inhibiting substance on isolated epithelial and mesenchymal cells during Müllerian duct regression in the rat. Endocrinology 131 1481-1488.

Wang PY, Protheroe A, Clarkson AN, Imhoff F, Koishi K \& McLennan IS 2009 Müllerian inhibiting substance contributes to sex-linked biases in the brain and behavior. PNAS 106 7203-7208. (doi:10.1073/pnas. 0902253106)

Wei X, Dombkowski D, Meirelles K, Pieretti-Vanmarcke R, Szotek PP, Chang HL, Preffer FI, Mueller PR, Teixeira J, MacLaughlin DT et al. 2010 Müllerian inhibiting substance preferentially inhibits stem/progenitors in human ovarian cancer cell lines compared with chemotherapeutics. PNAS 107 18874-18879. (doi:10.1073/pnas.1012667107)

Received in final form 6 March 2014

Accepted 12 March 2014

Made available online as an Accepted Preprint

12 March 2014
(C) 2014 Society for Endocrinology Printed in Great Britain 